Service social

\title{
Les demandes, les besoins et la planification des services aux personnes âgées
}

\section{François Béland}

Volume 34, numéro 1, 1985

Personnes âgées, milieux de vie et pratiques sociales

URI : https://id.erudit.org/iderudit/706248ar

DOI : https://doi.org/10.7202/706248ar

Aller au sommaire du numéro

Éditeur(s)

École de service social de l'Université Laval

ISSN

1708-1734 (numérique)

Découvrir la revue

Citer cet article

Béland, F. (1985). Les demandes, les besoins et la planification des services aux personnes âgées. Service social, 34(1), 14-29. https://doi.org/10.7202/706248ar d'utilisation que vous pouvez consulter en ligne.

https://apropos.erudit.org/fr/usagers/politique-dutilisation/ 
BÉLAND, François, chercheur au D.S.C., Cité de la santé de Ville-de-Laval et au Groupe de recherche interdisciplinaire en santé de la Faculté de médecine de l'Université de Montréal.

\section{Les demandes, les besoins et la planification des services aux personnes âgées}

\section{François Béland}

L'État s'est réservé un rôle de plus en plus actif dans le domaine des services sociaux et de santé depuis l'introduction de l'assurancehospitalisation et de l'assurance-maladie au Canada. Si l'État n'intervient pas au même titre que les professionnels pour définir les besoins des usagers, son rôle de planificateur, de gestionnaire et, à plusieurs titres, de financier du réseau, en fait un définisseur des besoins de la population. Ultimement, ces définitions sont des représentations que les organismes de planification se font de la situation qui les préoccupe.

Mais ce ne sont pas les seules qui soient valides, car les utilisateurs réels et potentiels des services se font, eux aussi, des représentations de leurs besoins qu'ils transforment en demande lorsqu'ils se présentent devant un professionnel de la santé et des services sociaux. Si une planification réussie implique que soient simultanément considérés les besoins déterminés par les professionnels et les demandes de la population, dans la pratique, il peut être extrêmement difficile de concilier les uns et les autres. Le processus de détermination des besoins par des méthodes dites scientifiques, avec recours à des professionnels, semble toujours détenir un privilège par rapport aux interprétations que fait le public de ses propres besoins et de leur traduction en demandes diverses.

Nous montrerons comment, dans un domaine qui semble superficiellement faire l'unanimité, celui des services et soins à domicile pour personnes âgées, les postulats les plus fondamentaux de ces programmes ne peuvent être soutenus en égard aux demandes des personnes âgées pour répondre à des besoins qui semblent devoir être 
satisfaits par les services à domicile. Cet exemple nous permettra de développer des commentaires plus généraux sur les rapports entre le point de vue de l'utilisateur et la détermination de leurs besoins par les organismes de planification.

\section{L'État, le marché et les besoins}

De façon traditionnelle, le consommateur satisfait ses besoins sur un marché, qui peut être défini, de façon très générale, par les relations d'échange entre deux agents: le consommateur et le pourvoyeur (Evans, 1981; Richardson, 1981). Dans le domaine de la santé, ces relations d'échange s'établissent entre plusieurs agents aux statuts variés: gouvernement, compagnies ou régie d'assurance, industries pharmaceutiques et d'équipements médicaux, agences professionnelles de régulation des praticiens, administration d'établissement où sont produits les actes professionnels, utilisateurs etc. (Evans, 1981). Les relations usuelles entre agents ne décrivent pas adéquatement les échanges directs ou indirects qui s'établissent dans ce marché spécifique (Donabedian, 1976; Evans, 1973; Stoddard et Barer, 1981). En particulier, les rapports de l'utilisateur aux techniques médicales et aux prescriptions pharmaceutiques se définissent par la présence d'intermédiaires socialement légitimes que sont les médecins et les autres professionnels. Le fait que les relations d'échange soient caractérisées par la présence de multiples agents qui entretiennent des rapports autant politiques, institutionnels, administratifs qu'économiques, rend problématique la traduction des besoins de l'utilisateur en demande de services.

Sur un marché classique, le point de vue de ce dernier se manifeste par ses achats. Dans le système médical, les professionnels ont un rôle fondamental dans l'interprétation des besoins, dans la détermination de la légitimité de l'utilisation et dans la somme et le type de services effectivement utilisés. Le rôle des agences gouvernementales est tout aussi important, du moins au Canada, où non seulement les professionnels sont rémunérés en grande partie par des honoraires négociés provincialement, mais où les établissements hospitaliers, les centres de soins prolongés, les centres d'hébergement à long terme et les unités de première ligne sont réglementés par les états provinciaux. Les responsabilités de planification, d'implantation et de gestion des ressources de santé définissent l'État comme un agent avec des intérêts spécifiques. Ce dernier intervient dans le rapport entre les besoins des utilisateurs et leur satisfaction et il se définit donc comme un autre des intermédiaires. 
Dans cette perspective, l'État recourt à la planification pour définir les grands axes de son intervention sur la base de sa compréhension des besoins de la population, des ressources dont il dispose et de son orientation idéologique. Qui plus est, il a la responsabilité d'arbitrer la distribution des ressources entre plusieurs domaines d'activités. De la même façon, il lui est loisible de combiner par des politiques et des programmes spécifiques des ressources de plusieurs secteurs (par exemple, l'habitation, le revenu, les services à domicile, les soins médicaux prolongés et les services sociaux dans le cadre d'une politique de maintien des personnes âgées dans la communauté), et de réinterpréter de cette façon les besoins de la population hors des champs traditionnels d'activités médicales ou sociales ou hors de marchés spécifiques. L'État peut ainsi substituer des formes d'aide à d'autres pour atteindre un certain degré d'efficacité ou pour briser des monopoles. De la sorte, le domaine des soins médicaux et son marché propre se voit investi par des ressources que ne contrôlent pas ses agents usuels.

Par son action, l'État entre en contact avec les utilisateurs des services de santé de façon indirecte. Il y a rencontre entre une offre planifiée de services et des demandes. Cette dernière notion n'est pas prise ici dans un sens strict. D'ailleurs, nous employons plutôt cette expression au pluriel, pour la distinguer de l'acception plus répandue chez les économistes. Nous traiterons ici de la question des demandes sous l'angle de leurs rapports avec la planification étatique et en tenant compte des débats idéologiques qu'elles peuvent susciter (Coward, 1979: Lakoff, 1976), ce qui sera illustré plus loin. De façon plus précise, à l'aide de l'exemple des soins et services à domicile, deux questions seront traitées: a) quel est l'effet potentiel de l'introduction des demandes des consommateurs dans la planification étatique des services sociaux et de santé aux adultes de 65 ans et plus ? et b) est-ce que les résultats d'études qui ont cherché à expliquer les demandes de certains services peuvent remplacer les mythes commodes qui servent souvent d'éléments idéologiques pour décider du développement ou de la décroissance des services de l'État?

\section{Deux études empiriques}

\section{a) Les besoins}

Une étude des besoins de services sociaux, médicaux et paramédicaux d'un échantillon d'adultes âgés de 65 ans et plus de la rive sud de l'île de Montréal a été entreprise en 1978 à l'aide du système 
C.T.M.S.P. ("Classification par type des malades en soins prolongés", Tilquin et al, 1977, 1980, 1981). L'échantillon a ici été pondéré pour représenter l'ensemble des adultes de 65 ans et plus du Québec.

Des équipes multidisciplinaires de professionnels ont déterminé les services requis par chacun des interviewés non institutionnalisés de l'échantillon de la rive sud de Montréal, demeurant dans des logements privés, sur la base des renseignements recueillis par les formulaires du C.T.M.S.P. En somme, l'étude de 1978 reproduit pour cet échantillon le processus de décision que les experts (travailleurs sociaux, infirmières, médecins, etc.) emploient actuellement dans plusieurs régions du Québec pour déterminer les besoins de leurs clients.

Les services requis par les personnes composant l'échantillon pondéré de 1978 ont été classés en quatre profils (Béland, 1983). Au tableau 1, les interviewés du premier type requièrent des soins médicaux généraux et des soins spécialisés ou une aide paramédicale. Ces individus, qui représentent $47.2 \%$ de l'échantillon, n'exigent aucun autre service pour assurer leur bien-être. En particulier, les transports étant exclus, ils peuvent utiliser les ressources disponibles à toute la communauté et ne requièrent aucun des services sociaux ou de santé du réseau des Affaires sociales du Québec au-delà de ceux disponibles à tous, ce qui est donc vrai pour la moitié de la population de 65 ans et plus. Celle-ci ne forme donc pas un groupe homogène, ni une population cible en soi. La fréquence de besoins de services n'est pas un critère suffisant pour constituer un groupe cible et les facteurs particuliers associés à cette fréquence doivent être identifiés pour cerner de façon systématique les groupes à risque dans une souspopulation. Une planification réaliste des services ne peut être faite sans ces renseignements vitaux.

Le profil B compte environ $37.3 \%$ des interviewés. Les services requis, tels que déterminés par l'équipe multidisciplinaire d'experts du C.T.M.S.P., se concentrent presque exclusivement autour des travaux lourds, des emplettes et de l'entretien ménager. Selon nos calculs, le tiers des personnes de ce groupe ne peuvent faire exécuter ces tâches. Par un programme relativement simple, une portion importante des besoins des individus de 65 ans et plus pourrait être satisfaite. II suffit de penser aux conséquences de l'absence d'aide pour les travaux lourds sur l'état du logement pour se convaincre de l'importance d'une telle initiative. Puisque l'incapacité d'entreprendre ces besognes peut être considérée comme la plus importante, un programme d'aide devrait comporter une composante préventive, le personnel étant bien placé pour surveiller l'évolution de la fonctionnalité des clients desservis. Cette forme d'aide doit donc être coordonnée aux autres 
TABLEAU 1

Nombre moyen d'unités de services requis par des adultes de 65 ans et plus au Québec, 1978, selon le système C.T.M.S.P.

\begin{tabular}{|c|c|c|c|c|c|}
\hline \multirow{2}{*}{ Services requis } & \multirow{2}{*}{ Unités de mesure } & \multicolumn{4}{|c|}{ Profil } \\
\hline & & A & B & C & D \\
\hline \multicolumn{6}{|l|}{ Soins infirmiers } \\
\hline -court terme & Heures par jour & - & .02 & .01 & $.42^{*}$ \\
\hline -long terme & Heures par jour & - & .01 & .04 & $.18^{*}$ \\
\hline \multicolumn{6}{|l|}{ Services sociaux } \\
\hline -court terme & Heures par jour & .05 & .06 & $.41^{*}$ & $.21^{*}$ \\
\hline -long terme & Heures par jour & .11 & .06 & .04 & $.12^{*}$ \\
\hline Ergothérapie et physiothérapie & Heures par semaine & .09 & .04 & .40 & $.92^{*}$ \\
\hline Services médicaux & Nombre par année & $3.31^{*}$ & $4.24 *$ & $4.75^{*}$ & $5.00^{*}$ \\
\hline Services paramédicaux et soins & & & & & \\
\hline spécialisés & Nombre par année & $.37^{*}$ & $.60^{*}$ & $.73^{*}$ & $.69^{*}$ \\
\hline \multicolumn{6}{|l|}{ Activités de la vie quotidienne } \\
\hline -préparation des repas & Nombre par semaine & - & .30 & $14.80^{*}$ & $15.61^{*}$ \\
\hline -emplettes et entretien & Nombre par mois & .08 & .80 & $3.49 *$ & $4.75^{*}$ \\
\hline -travaux lourds & Nombre par année & .47 & $6.60^{*}$ & $7.42^{*}$ & $7.75^{*}$ \\
\hline -transport & Type: normal $=0 ;$ spécialisé $=1$ & .01 & .07 & .24 & $.56^{*}$ \\
\hline -surveillance & Heures par jour & - & - & .74 & $7.50^{*}$ \\
\hline -amélioration des relations sociales & Heures par jour & - & .43 & $.71^{*}$ & .17 \\
\hline
\end{tabular}

* Services requis dans un profil. 
programmes et aux services professionnels disponibles dans la communauté.

Les personnes de la troisième catégorie (6,0\% de l'échantillon) requièrent une assistance beaucoup plus élaborée pour l'organisation de la vie quotidienne. Ce groupe se caractérise par la conjonction de besoins de préparation des repas, d'emplettes et d'entretien ménager et de travaux lourds, en plus des services médicaux traditionnels.

Au profil D, l'ensemble des services énumérés au tableau 1 sont requis. Ici, les interviewés représentent près de $9,5 \%$ de la population des 65 ans et plus du Québec qui vivent dans un logement privé. L'aide requise est extrêmement lourde à supporter et semble indiquer qu'une proportion importante d'entre eux pourraient être institutionnalisés.

Mais d'autres données contenues dans les fichiers du système C.T.M.S.P. montrent que presque toutes les personnes du profil D habitent soit chez un enfant, soit chez un membre de leur parenté. Presque la moitié des interviewés ou leurs conjoints ayant été administrateurs ou professionnels avant la retraite se retrouvent dàns ce profil, et aucun des individus de ces deux catégories d'occupation ne sont classés dans C. La disponibilité de l'aide familiale est intimement liée aux ressources familiales plutôt qu'à l'éclatement de cette institution sociale.

Les personnes du profil C vivent une situation difficile: ce sont surtout des femmes seules et âgées qui doivent compter sur du support extérieur pour satisfaire les besoins de base: préparation des repas, emplettes et entretien de leur logement. De même, puisque les hommes seuls de 65 à 74 ans ne sont pas représentés dans l'échantillon, alors qu'ils constituent 7,5\% de la population des hommes de 65 ans et plus au Québec, nous sous-estimons de façon importante les besoins totaux de services du profil C.

Les adultes âgés, affectés des incapacités fonctionnelles les plus lourdes et qui vivent dans la communauté, sont donc habituellement abrités par leur famille, qui disposerait de ressources économiques et matérielles à la mesure des responsabilités qu'elle assume. Les personnes de ce groupe d'âge qui ne sont pas aussi favorisées (celles du profil C, en particulier) ne disposent que des ressources collectives d'hébergement. Les critiques de l'institutionnalisation devraient prendre en compte la situation qui a imposé cette solution; les comparaisons entre personnes institutionnalisées et celles maintenues à domicile seraient alors moins défavorables à l'institution (Myles, 1978, 1979).

Deux mythes sont largement répandus quant à la situation familiale et aux préférences de la population étudiée: les familles n'aident 
plus leurs membres âgés (Shanas, 1979) et ces adultes préfèrent demeurer dans leur milieu naturel (American Nurses' Association, 1978; Bell, 1973 ; Berg et al., 1974 ; Brickner, 1976; Ministère des affaires sociales, 1979; Massachusetts Department of Elder Affairs, 1976). II est clair, d'après les caractéristiques socio-économiques et socio-démographiques des personnes du profil $D$, que la famille est une institution encore extrêmement active auprès de ses membres âgés et ce, d'une façon traditionnelle, en les abritant. L'autre mythe ne peut se transformer en savoir relativement fondé qu'en demandant l'avis des premiers concernés.

Les données que nous venons de dévoiler sont riches. Nous ne pouvons ici les exploiter telles qu'elles le méritent. Mais remarquons, pour les fins de la discussion, que les besoins de services des personnes du profil C sont habituellement offerts par tous les bons programmes de services à domicile. Dans la mesure où c'est le mythe soutenant que les adultes de 65 ans et plus veulent demeurer à domicile qui préside aux décisions bureaucratiques quant au développement de programmes, l'existence de cette catégorie est une indication que les services à domicile doivent devenir une priorité.

\section{b) Les demandes}

Quelles sont les préférences résidentielles des adultes de 65 ans et plus? Une étude de trois échantillons aléatoires simples d'adultes de ce groupe (tableau 2) pris dans deux villes de taille moyenne du Québec et dans un quartier ouvrier de Montréal (Béland, 1980), montre, d'une part, que des demandes formelles d'hébergement de toutes formes (de la résidence réservée aux personnes âgées jusqu'à l'institution médicale) effectivement déposées auprès des établissements sont de $10 \%$ de l'échantillon total. D'autre part, $53.2 \%$ des interviewés ont exprimé le désir de quitter leur domicile pour une autre forme d'habitation. En effet, $27.4 \%$ désiraient habiter une résidence pour personnes âgées, entre $10.8 \%$ et $13.2 \%$ dans un centre d'accueil et d'hébergement pour personnes âgées. Dans l'échantillon 3, 12.6\% exprimaient le désir de s'installer à demeure dans un hôpital; ce que nous ne pouvons expliquer par l'état des ressources socio-sanitaires de ce territoire.

Après analyse, Béland (1981, 1984a, 1984b) a émis ces principaux facteurs d'explication : la présence de plusieurs maladies chroniques; un inconfort psychologique important ; l'absence d'aide disponible de la part d'un co-résident; le fait de vivre seul ou avec un époux seulement plutôt qu'avec un membre de la famille ou un ami; et les conditions physiques du logement. Or, il y a une correspondance qui 
TABLEAU 2

Les demandes et les désirs d'hébergement dans trois échantillons d'adultes de 65 ans et plus au Québec

\begin{tabular}{|c|c|c|c|c|c|c|}
\hline \multirow{2}{*}{ Hébergement } & \multicolumn{2}{|c|}{ Échantillon 1} & \multicolumn{2}{|c|}{ Échantillon 2} & \multicolumn{2}{|c|}{ Échantillon 3} \\
\hline & $N$ & $\%$ & $\mathrm{~N}$ & $\%$ & $\mathrm{~N}$ & $\%$ \\
\hline \multicolumn{7}{|l|}{ Demandes } \\
\hline Non & 236 & 83.1 & 280 & 83.6 & 332 & 87.4 \\
\hline Oui & 32 & 11.3 & 34 & 10.1 & 32 & 10.0 \\
\hline Déjà en résidence privée & 16 & 5.6 & 21 & 6.3 & 10 & 2.6 \\
\hline \multicolumn{7}{|l|}{ Désirs } \\
\hline $\begin{array}{l}\text { Dans un centre hospitalier de } \\
\text { soins prolongés }\end{array}$ & 17 & 5.2 & 23 & 6.9 & 98 & 12.6 \\
\hline Dans un centre d'accueil & 41 & 12.5 & 36 & 10.8 & 50 & 13.2 \\
\hline Dans une conciergerie spécia- & & & & & & \\
\hline lisée pour personnes âgées & 78 & 23.8 & 79 & 23.7 & 104 & 27.4 \\
\hline Aucun choix & 192 & 58.5 & 195 & 58.6 & 178 & 46.8 \\
\hline
\end{tabular}

n'est probablement pas fortuite entre les besoins de services des personnes du profil $\mathrm{C}$ et les caractéristiques sociales et psychosociales de celles qui désirent quitter leur domicile ou qui ont déjà déposé une demande d'hébergement auprès d'institutions privées ou publiques de toutes catégories.

Une des hypothèses pour expliquer ces désirs et demandes pourrait se formuler ainsi : une bonne proportion des personnes du profil C désirent quitter leur domicile pour un lieu où l'aide qu'elles requièrent sera immédiatement disponible. II est facile d'évacuer le problème en parlant de sentiment d'insécurité ; mais le problème n'est pas tellement celui de l'insécurité comme celui des exigences des personnes âgées pour la réduire. Cette interprétation est appuyée par la corrélation entre l'absence d'une source d'aide résidant à domicile et les désirs de le quitter (Béland, 1981), et aussi par le fait que les personnes très âgées fonctionnellement handicapées et atteintes de plusieurs maladies chroniques qui habitent avec un de leurs enfants ou un parent n'éprouvent pas ce désir de quitter leur domicile (Béland, 1984b).

Une interprétation des besoins de services contenus dans le profil $C$, à la lumière du mythe selon lequel les adultes de 65 ans et plus désirent demeurer à leur domicile, mène à l'organisation de services à domicile; de la même façon qu'une vision mécaniciste de la planification conclurait à la nécessité de mettre à la disposition de ces 
personnes cette forme d'assistance particulière, et celle-là seulement, de la façon la plus économique. Par ailleurs, une interprétation qui admet que les conditions de vie de ces gens les amènent à une demande légitime de relocalisation exige le développement d'habitations protégées de diverses formes; tandis que les services à domicile peuvent être conçus comme une aide d'appoint disponible aux résidents de ces habitations.

\section{La planification gouvernementale et les besoins des malades chroniques}

\section{a) Des divergences d'interprétation}

Chacun des profils de besoins de services du tableau 1 est identifié par l'intermédiaire d'une procédure formelle, qu'il est possible de reproduire par d'autres études qui emploieraient la même méthodologie. Il est probable qu'une méthode d'estimation différente du C.T.M.S.P. (Kane et Kane, 1981), mais qui vise les mêmes buts, produira les mêmes résultats. Cependant, il existe des traductions possiblement divergentes des besoins en offre de services. Ces difficultés sont particulièrement évidentes à propos du profil C. Une perspective courante, de nos jours, y voit une confirmation de ses orientations : les services à domicile assumeront la responsabilité de satisfaire des besoins pour lesquels ils ont été pensés en tout premier lieu puisque nous avons pu établir que : la population-cible visée par cette forme d'aide existe ; qu'elle est identifiée par des caractéristiques spécifiques ; et que son volume est proche de celui habituellement fixé, soit plus ou moins $5 \%$ de la population âgée non institutionnalisée. Pourtant, les caractéristiques qui identifient la clientèle des services de soins à domicile (S.S.D.) sont les mêmes que celles qui prédisent les désirs et les demandes d'hébergement, auprès d'un échantillon d'adultes de 65 ans et plus du Québec, et ce malgré la présence d'une aide semblable à celle des services à domicile.

Il n'y a ici aucun arbitre, fût-il professionnel de la santé ou des services sociaux, pour légitimer l'une ou l'autre de ces deux perspectives. En effet, le domaine d'interprétation des besoins des personnes de 65 ans et plus affectés par des maladies chroniques, des incapacités fonctionnelles et des pertes successives d'êtres chers se définit d'emblée comme plus grand que celui des personnes de tout âge qui souffrent de maladies aiguës, mais guérissables, selon les standards de la médecine contemporaine. Puisque, dans ce dernier cas, la société reconnaît la légitimité du régime de traitement médical, le malade sera 
plus que moins contraint d'adopter le rôle de malade (PIfanz et Keupp, 1977), donc de s'adresser au corps médical pour obtenir une guérison. Aussi, les pertes de fonctionnalités physiques ou sociales sont médiatisées par les capacités et les ressources personnelles disponibles. Si le régime de traitement professionnel, souvent inexistant de toute façon pour les maux chroniques physiques ou sociaux, peut être contraignant, le malade chronique ou la personne isolée ne se voit pas désigner un rôle social unique défini en fonction des actions thérapeutiques ou de réhabilitation qui leur sont prescrites (Gallagher, 1976). La recherche de solutions à la perte de fonctionnalités physiques et sociales ne repose donc pas sur des prescriptions professionnelles encadrées par une connaissance ou un art clinique plus ou moins cristallisé.

\section{b) La planification}

Les planificateurs des organismes gouvernementaux préfèrent la notion de besoins à celle de demandes, car celles-ci sont des comportements et, à ce titre, difficiles à prévoir, à saisir, à régulariser et à contrôler. Cette notion implique que le consommateur reste libre et presque insoumis aux prescriptions et pressions bureaucratiques, privées ou publiques, aussi bombardé soit-il par les messages biaisés ou par de multiples canaux d'information, et aussi livré soit-il à des influences diverses, allant du climat socio-culturel général d'une société jusqu'à celles de la personne la plus proche. La notion de besoins semble par ailleurs plonger dans les racines les plus fondamentales des nécessités de la vie. Si celles-ci ne sont pas satisfaites, l'être humain est en deçà de sa propre dignité. Ce concept a un air de nécessité que la demande n'a pas, mais sa beauté vient de sa capacité à légitimer le travail de planification.

Les besoins, une fois déterminés par des méthodes scientifiques, permettent d'élaborer des plans d'action appelés à les satisfaire. Ils sont le fondement même de la rationalité bureaucratique (une expression que nous employons sans sous-entendu malveillant). Tous les cours universitaires de planification insistent sur la nécessité de fonder les activités d'un réseau de soins de santé et de services sociaux sur des études scientifiques des besoins. En santé, l'ouvre de Donabedian $(1973,1976)$ a presque marqué un point de rupture à cet égard, en systématisant la procédure de planification des services médicaux, mais tout en proposant une éthique respectueuse des droits égaux à des services accessibles et de qualité pour tous. 
Mais les besoins n'existent pas dans un vide social absolu où l'expertise de quelques-uns permettrait de les classifier dans des catégories exhaustives et mutuellement exclusives, prêtes pour l'élaboration de plans, de programmes et d'activités judicieuses, économiques et de qualité. Il y a toujours des choix normatifs et éthiques dans les études de besoins, ne serait-ce qu'en fonction du rôle des experts qui les évaluent sur la base des données recueillies.

Dans la mesure où une société reconnaît la légitimité de l'expertise d'individus ayant acquis une formation spécialisée, les études des besoins peuvent recourir à celle-ci pour reproduire les décisions qui se prennent quotidiennement dans un système de distribution des soins médicaux et de services sociaux. L'état des besoins d'une population, tel que déterminé au cours d'une étude, permet de comparer les capacités des ressources existantes à celles requises pour en satisfaire l'ensemble, donc, à prendre des décisions éclairées sur l'orientation future des efforts publics ou privés.

La décision bureaucratique de satisfaire des besoins non comblés et les modalités des actions à entreprendre pour ce faire seront directement inspirées des résultats des études dans un processus de planification qui entend respecter les exigences fondamentales de la rationalité la plus élémentaire. Mais, si ces études sont entachées de choix normatifs en fonction du régime de valeurs qui préside à leur détermination, la décision de satisfaire tel besoin plutôt que tel autre et les modalités pour le faire sont des choix politiques, qui ne pourront jamais être déterminés exclusivement par un processus s'appuyant sur une démarche scientifique.

Les limites au processus de rationalité importent cependant moins que la sélection des critères qui guident l'action dans un contexte donné, mais elles permettent aux préférences, aux normes et aux valeurs de s'introduire : les bureaucraties publiques ont donc au moins le choix de décider en fonction de leurs intérêts propres ou d'introduire aussi dans leurs décisions les préoccupations et les préférences des gens qu'elles ont le devoir de desservir dans le cadre de mandats généraux ou spécifiques définis par les États canadiens provinciaux ou fédéral. En un mot: les bureaucraties ont le loisir d'intégrer ou non la notion de demandes dans l'arsenal conceptuel de leur processus de décision.

\section{Discussion}

Nous avons illustré comment, au niveau de la planification, des décisions peuvent être orientées par des mythes ou par les résultats 
d'étude des demandes et des désirs de la population. Le point de vue du consommateur est plus qu'un ensemble de réclamations et de récriminations vis-à-vis un appareil puissant. II est avant tout une proposition de solution à des problèmes vécus. Il entraîne des comportements qui ont non seulement un impact sur l'état des ressources et des services gouvernementaux et privés mais qui sont des éléments fondamentaux du processus de planification lui-même puisqu'il fixe des limites à son efficacité, propose des solutions concurrentes ou convergentes aux mêmes problèmes et repose continuellement la question des valeurs et des orientations normatives.

La planification, lorsqu'elle repose sur des études ou des estimations des besoins, se base la plupart du temps sur des jugements professionnels dont la légitimité est reconnue dans notre société. Mais, si cette démarche privilégie des valeurs et des normes, il est illusoire d'assumer qu'il y a un rapport automatique entre l'estimation des besoins de services et les programmes qui les satisfont. Les premiers se décomposent en unités de services qui se regroupent en unité administrative de façon très diverse et sont assurés à l'intérieur de contextes variés : du domicile privé à l'institution la plus réglementée.

L'incertitude des processus de décisions peut être camouflée par la "scientificité" apparente des analyses et recherches. Mais, dans la réalité des choses et événements, les utilisateurs potentiels exercent des pressions soit en utilisant des services pour d'autres fins que celles prévues par les planificateurs, soit en se retirant des programmes offerts. Ultimement, la pression sera d'ordre politique. Le point de vue du consommateur n'est donc pas qu'une réclamation, une protestation, mais il se manifeste quotidiennement dans l'administration des ressources de services sociaux et de santé. Il doit donc être inclus dans le processus de planification même par un ensemble de moyens qui vont de la représentation politique jusqu'au souci d'intégrer les demandes dans les études scientifiques.

Les besoins ne sont jamais que des descriptions valorisées par le jugement de ceux qui les estiment alors que les demandes sont des principes actifs qui font pression sur les ressources selon l'utilisation qu'elles commandent. Dans le domaine de la santé, les professionnels sont une source importante de la demande de services par leurs prescriptions (Evans, 1973; Wilensky et Rossiter, 1981). Mais, les conséquences des maladies chroniques sur les conditions de vie des patients, tout aussi bien que le régime des traitements professionnels, ont un impact considérable sur l'état d'un système de soins médicaux et de services sociaux. Le consommateur est en mesure de juger de la 
qualité des rapports entre ses besoins personnels, les ressources offertes dans la communauté et celles qu'il désire mobiliser.

L'exemple des services et soins à domicile est éclairant à cet égard lorsque les besoins des personnes classées au profil C sont comparés à l'assistance offerte par leurs réseaux sociaux informels, par les programmes de services et de soins à domicile et aux ressources d'hébergement. En effet, cette troisième catégorie identifie des personnes qui sont affectées par des incapacités fonctionnelles graves et qui vivent une situation difficile : elles sont seules ou partagent leur vie avec une personne du même âge sur laquelle repose la responsabilité d'une lourde tâche. Leurs souhaits de relocalisation sont rationnels en rapport à leurs conditions de vie. La situation des personnes classées au profil $D$ est tout autre puisque leurs familles assurent l'essentiel de leurs besoins. Dans ce contexte, les services à domicile sont appelés à jouer un rôle d'appoint à celui de la famille. Mais leur assigner le but de maintenir à domicile les personnes du profil C relève de la magie ou de la témérité (Béland, 1984a ; Dunlop, 1980). Il est donc différent d'assumer que les services à domicile sont un substitut à des demandes de relocalisation et de compter sur eux pour permettre aux personnes le désirant de demeurer chez elles.

\section{Conclusion}

La détermination des besoins est un stade assez primaire, mais fondamental, dans le processus de planification bureaucratique des services et dans le processus individuel de la formation des demandes. Puisqu'il existe des degrés de liberté entre ces deux notions, puisque le processus de planification peut être conçu comme la traduction technocratique des besoins en demandes socialement légitimes, puisque au moins deux interprétations des dernières s'opposent : celle des planificateurs et celle des consommateurs, quoique l'interprétation des professionnels s'ajoute aussi à ce diptyque, la question du pouvoir de légitimation des besoins se pose avec acuité.

Dans la pratique, les considérations normatives sur la légitimité n'occupent pas une place centrale chez les planificateurs, les professionnels et les consommateurs. Heureusement, sinon les débats mobiliseraient les ressources nécessaires à la production de services. Mais, il faut examiner périodiquement les références idéologiques implicites des pratiques. Jusqu'ici, la notion de besoins a dominé le champ de la planification comme si elle réussissait à en fonder la légitimité, laissant peu de place à la notion de demandes. Dans ce contexte, les consommateurs sont appelés à se référer aux premiers et 
à les définir de la même manière. Ce qui est acceptable dans la mesure où il est bien compris que la distance entre besoins et demandes est grande et laisse place à l'introduction de valeurs et d'orientations normatives. Dans un contexte où les demandes de services sont prises en compte dans la planification, il n'y a plus de solution unique aux besoins de la population, mais une diversité de moyens qui correspondent aux multiples façons rationnelles dont les premiers concernés choisissent de solutionner leurs problèmes.

II ne s'agit pas de rendre la rationalité bureaucratique victime des demandes de la clientèle potentielle, dans la même mesure où l'inverse se vérifie peut-être aujourd'hui. Il s'agit plutôt de reconnaître qu'il y a des circonstances qui rendent légitimes certains choix. Ici aussi, nous sommes pleinement dans le domaine des valeurs et des orientations normatives, non pas pour quitter le domaine de la rationalité mécaniciste, mais bien pour reconnaître le rôle de l'incertitude dans toute décision de planification et dans tout travail d'orientation de politique. Il est aisé de disqualifier les résultats de recherche sur les besoins en introduisant des considérations diverses, fondées ou non, sur la demande populaire et sur son caractère insaisissable. En fait, la planification ne vise ni à maximiser ni à minimiser la "scientificité " des résultats de recherche, ni l'incertitude face à des choix normatifs. Elle est une activité qui se fonde sur l'un et l'autre domaine, et sa tâche est de les harmoniser pour aboutir à des propositions concrètes qui sauront prendre en compte la diversité des situations vécues par les personnes dont elle a la mission de faciliter la vie.

\section{Références bibliographiques}

American Nurses' Association, Health Care at Home : Essential Component of a National Health Policy, Kansas City, American Nurses' Association, 1978.

BélAND, F., Méthodologie pour l'évaluation des programmes socio-sanitaires: le cas des services à domicile pour personnes âgées, Québec, Laboratoire de recherches sociologiques, Université Laval, cahier no 15, 1980.

BÉLAND, F., "L'aide aux personnes âgées, leurs incapacités fonctionnelles et leurs désirs d'hébergement: les résultats d'une étude empirique", dans: C. Tilquin, Science des systèmes dans le domaine de la santé, Toronto, Pergamon, 1981.

BÉLAND, F., "Application of the CTMSP system to a non-institutionalized elderly population : identifying profiles of service requirements", texte présenté à la Conférence Systed' 83, Montréal, 1983. 
BÉlAnd, F., "The decision of elderly to leave their homes", The Gerontologist, 24, 1984a : 179-185.

BÉLAND, F., "The family and adults 65 years of age and over: coresidency and availability of help ", Canadian Review of Sociology and Anthropology, 23, 1984b : 302-317.

BÉLAND, F., "L'évaluation de la politique des services à domicile du Ministère des affaires sociales du Québec: une étude de cas", Revue canadienne du vieillissement, vol. 3, no 4, 1984c: 175-192.

BELL, W.G., "Community care for the elderly: an alternative to institutionalization ", The Gerontologist, 13, $1973:$ 344-354.

BERG, R.L., L. AtLAS et J. ZEIGER, "Integrated homemaking services for the aged in urban neighborhoods", The Gerontologist, 14, 1974 : 388-393.

BRICKNER, P.W., "Home maintenance for the home-bound aged : a pilot program in New York City", The Gerontologist, 16, 1976: 25-59.

CoWARD, R.T., "Planning community services for the rural elderly: implications from research ", The Gerontologist, 19, 1979: 275-282.

Donabedian, A., Aspects of Medical Care Administration, Cambridge, Harvard University Press, 1973.

Donabedian, A., Benefits in Medical Care Programs, Cambridge, Harvard University Press, 1976.

DUNLOP, B., "Expanded home-based care for the impaired elderly : solution or pipe-dream ", American Journal of Public Health, 70, 1980 : 514-519.

Evans, R., "Supplier-induced demand: some empirical evidence and implications", dans: M. PERLMAN, The Economics of Health and Medical Care, New York, Wiley, 1973: 162-173.

Evans, R., "Incomplete vertical integration: the distinctive structure of the health-care industry", dans: J. VAN der GAaG et M. PerLman, Health, Economics, and Health Economics, Amsterdam, North-Holland, 1981: 329-354.

GallaGher, E., "Lines of reconstruction and extension in the Parsonian sociology of illness", Social Science and Medicine, 10, 1976: 207-218.

KAne, R.A. et R.L. KAne, Assessing the Elderly: A Practical Guide to Measurement, Lexington, Lexington Books, 1981.

LAKOFF, S.A., "The future of social intervention", dans: R.H. BINATOCK et E. ShanAs, Handbook of Aging and the Social Sciences, New York, Van Nostrand Reinhold, 1976:643-663.

Ministère des affaires sociales, Les services à domicile : politique du Ministère des affaires sociales, Québec, Ministère des affaires sociales, 1979.

Massachusetts Department of Elder Affairs, Home Care: An Alternative to Institutionalization, Boston (Mass.), Massachusetts Department of Elder Affairs, National Technical Information Service, 1976.

MyLES, J., "Institutionalization and sick role identification among the elderly", American Sociological Review, 43, 1978 : 508-521.

MrLEs, J., "Institutionalization and disengagement among the elderly", Canadian Review of Sociology and Anthropology, 16, 1979: 171-182. 
Plfanz, M. et H. KeupP, "Le concept de maladie : une perspective sociologique", Revue internationale des sciences sociales, 24, 1977 : 415-427.

RICHARDSON, J., "The inducement hypothesis: that doctors generate demand for their own services", dans : J. VAN Der GAaG et M. PERLMAN, op. cit. : 189-214.

SHANAS, E., "Social myth as hypothesis: the case of the family relations of old people", The Gerontologist, 19, 1979 : 3-9.

Stoddard, G. et M.L. BARER, "Analysis of demand and utilization through episodes of medical services", dans: J. VAN der GAaG, et M. Perlman, op. cit. : 149-170.

TILQUIN, C. et al., "Administration d'un réseau de services socio-sanitaires pour les personnes âgées", Administration hospitalière et sociale, 23, 1977 : 26-32.

TILQUIN, C. et al., "The physical, emotional and social conditions of an aged population in Quebec", dans: V.MARSHall, Aging in Canada, Toronto, Fitzhenry and Whiteside, $1980: 222-231$.

TILQUIN, C. et al., CTMSP 81: l'évaluation des services requis et la mesure des ressources requises par le bénéficiaire, Montréal, Université de Montréal, Équipe de recherche opérationnelle en santé, 1981.

WILENSKY, G. et L.F. Rossiter, "The magnitude and determinants of physician initiated visits in the United States", dans : J. VAN DER GAAG et M. PERLMAN, op. cit. : 215-244. 